\title{
Editorial
}

\section{Cervical strain/sprain (is this a metaphor or a medical diagnosis?)}

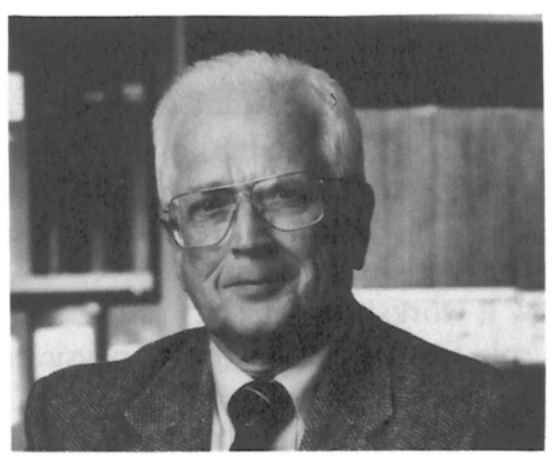

\section{Ernest W. Johnson}

Department of Physical Medicine and Rehabilitation, The Ohio State University, 370 West 9 th Avenue, Columbus, OH 43210-1238, USA

8470. This is an ICD-9 code? Shorthand for pain in the neck is more realistic.

Facet synovitis; occipital nerve neuritis; or myofascial pain uncovered by trauma. In the old days we heard 'whiplash' - an unfortunate term implying mode of injury, not result! To accept terminology such as 'whiplash', one would also accept 'fall from a ladder', 'slipped on stairs', 'moving a sofa', 'fell off a house', etc., etc.

But cervical sprain/strain is still meaningless - and fast becoming a metaphor for continued complaints... and TREATMENT.

I recently reviewed a patient's chart with this diagnosis who was still receiving twice weekly ultrasound, 'myofascial release', and acupuncture.

Is this really necessary?

To this question, I respond with an emphatic NO!

Not even nearly appropriate. But, should I indict the provider or the patient or both? 
Unfortunately, this is not an infrequent case ... but rather a typical one and a frequent example of overuse, or even abuse of health care services.

The clinician-provider appealed my denial and upon further review of the file, there were no objective neurologic signs or abnormal tests reported.

So this is simply a person c/o discomfort; such a complaint converted into medical gobbledegook to rationalize expensive physical treatment. And, incidentally, converting the insurer into a 'bad boy' for denying reimbursement.

What are reasonable options for management of this case?

I would support 6-8 sessions with a knowledgeable practitioner to evaluate the response to measures which could be used in a home program, e.g. heat/cold/exercise/TENS. This would be followed by a re-evaluation of response to a precisely prescribed home program at appropriate intervals - weekly for 3-4 weeks if acute, or every 2-3 months if long term.

The notion that any therapeutic benefit will accrue from once or twice weekly therapy in these musculoskeletal pain problems is incredibly naive or subtly Machiavellian.

It used to be axiomatic that 50 patients of this ilk could be a career for some practitioners - at least in an economic sense.

NB. Insurers will be critically reviewing irrational modes and durations of treatment. No longer will a physician scratching on a prescription form be sufficient to initiate and prolong physical treatment progress toward specific goals will be absolutely required.

Also necessary is some evidence (RCT?) in the peer-reviewed literature that a reasonable expectation of a favorable outcome will ensue after a recommended course of treatment.

Anecdotal testimonials will NOT be sufficient.

A practitioner's avowal of 'I know it works' won't do it!

I see in the future an increasing role for physiatrists, other physicians, as well as ancillary practitioners to be instructors to their patients (I avoid the pejorative term 'client') instead of hands-on curers.

For most muscular pain, Mother Nature (politically correct) plus adequate tincture of time and accompanied by the correctly prescribed active exercise (movement, not rest) is the winning combination. 